\title{
Overall survival after reirradiation of spinal metastases - independent validation of predictive models
}

\author{
Daniel Buergy ${ }^{*}$, Lena Siedlitzki, Judit Boda-Heggemann, Frederik Wenz and Frank Lohr
}

\begin{abstract}
Background: It is unknown if survival prediction tools (SPTs) sufficiently predict survival in patients who undergo palliative reirradiation of spinal metastases. We therefore set out to clarify if SPTs can predict survival in this patient population.

Methods: We retrospectively analyzed spinal reirradiations performed ( $n=58,52$ patients, 44 included in analysis). SPTs for patients with spinal metastases were identified and compared to a general palliative score and to a dedicated SPT to estimate prognosis in palliative reirradiation independent of site (SPT-Nieder).

Results: Consistently in all tests, SPT-Nieder showed best predictive performance as compared to other tools. Items associated with survival were general condition (KPS), liver metastases, and steroid use. Other factors like primary tumor site, pleural effusion, and bone metastases were not correlated with survival. We adapted an own score to the data which performed comparable to SPT-Nieder but avoids the pleural effusion item. Both scores showed good performance in identifying long-term survivors with late recurrences.

Conclusions: Survival prediction in case of spinal reirradiation is possible with sufficient predictive separation. Applying SPTs in case of reirradiation helps to identify patients with good life expectancy who might benefit from dose escalation or longer treatment courses.
\end{abstract}

Keywords: Palliative radiotherapy, Spinal metastases, Survival score, Reirradiation

\section{Background}

Survival from most types of cancer has increased steadily during the last years in developed countries [1]. Longer survival time is associated with an increased number of patients who live long enough to experience a recurrence after an initial radiotherapy [2]. Currently around $8-20 \%$ of patients with bone metastases receive reirradiation [3]. Although reirradiation is possible with modern techniques, it can be associated with an increased risk of side effects, depending on reirradiation site, volume, cumulative dose, fraction dose, and interval between irradiation and reirradiation [4-6]. Due to potentially debilitating side effects such as radiation myelopathy (RM) or radiation-induced sacral (plexus) neuropathy [7-9], historically only few studies of conventional reirradiation

\footnotetext{
* Correspondence: daniel_buergy@medma.uni-heidelberg.de Department of Radiation Oncology, Universitätsmedizin Mannheim, Medical Faculty Mannheim, Heidelberg University, Mannheim, Germany
}

of spinal metastases have been reported [7, 10-12]. A retrospective analysis of cases treated at the Mayo Clinic between 1975 and 1992 showed that 5 out of 54 patients developed neurologic deterioration after reirradiation. Although only one of them developed the typical BrownSequard syndrome, the authors could not determine if the other four patients had delayed RM, or if symptoms were triggered by spinal cord compression. Median time of onset for deterioration was 7.8 months, and median survival of all patients was 4.2 months, therefore it is possible that the number would have been higher, had patients survived longer [7]. Introduction of modern techniques such as Intensity Modulated Radiation Therapy (IMRT) or Stereotactic Body Radiation Therapy (SBRT) changed the approaches to reirradiation of paraspinal tumors profoundly. Today, as reviewed by Kirkpatrick et al. [13], multiple series on reirradiation of the spinal cord have been reported with low, albeit not completely absent risk 
of RM. The residual risk of RM in primary SBRT or reSBRT has been primarily attributed to higher doses per fraction [14]. On the other hand, new treatment concepts such as multiple ablative treatments in patients with oligometastatic disease warrant higher doses to obtain sufficient tumor control [15]. If patients benefit from longer, potentially more wearisome radiation courses, depends mainly on their life expectancy. It is unknown if available survival prediction tools (SPTs) can be applied to accurately predict survival in patients who are eligible for reirradiation of spinal metastases.

This study intends to validate available approaches/ instruments to predict survival in a patient group who underwent reirradiation for spinal metastases in a single institution.

\section{Patients and methods}

From November 2006 to July 2013, 58 spinal metastases were reirradiated in 52 patients. If a patient received a second reirradiation $(n=7)$, only the first reirradiation was included in further analysis. Three patients were excluded because they had received radiotherapy due to hematologic malignancies, or spinal ependymoma. Additionally, 4 patients were excluded because of missing data. The remaining patients $(n=44)$ were included in further analysis. Patient characteristics are detailed in Table 1 . The study was approved by the ethics committee of Heidelberg University, Medical Faculty Mannheim (2013-602N-MA).

Survival scores were identified by a literature search using PubMed with the following search terms: Spinal [OR] Spine [AND] Metastases Survival Score. Instruments designed for specific cancer entities [16] or for patients who already had spinal cord compression $[17,18]$ were excluded. SPTs which evaluated other outcomes than survival [2] were also excluded. Studies detailing SPTs that did not provide a numerical score to rank patients according to risk were transformed into such a scoring system, e.g. subgroups of "favorable, moderate, and unfavorable" were transformed into numerical values to facilitate nonparametric analysis. If such transformation was not reasonably possible, SPTs were excluded [19]. In addition to spine-specific scores, we included the SPT as developed by Chow et al. [20, 21] (SPT-Chow). SPT-Chow was chosen because it is a simple and validated tool, widely applicable in palliative settings [22]. Furthermore, we included the SPT developed for reirradiation in general by Nieder et al. [23]. SPTs as developed by Tokuhashi [24], Balain [25], Bauer [26], Bollen [27], and Tomita et al. [28], were deemed appropriate. Balain et al. called their tool the Oswestry risk index (SPT-Oswestry). All other SPTs were named according to the $1^{\text {st }}$ author of the article describing the index. All details on the SPTs are summarized in Additional file 1: Table S1. Each SPT was then applied to each patient, thereby assigning all patients to prognostic groups as defined by each SPT. We used different approaches to validate each prognostic model. First, we calculated a simple index of separation (PSEP), as defined by Altman et al. [29]. Basically, PSEP is the difference between $\mathrm{P}_{\text {worst }}$ and $\mathrm{P}_{\text {best }}$, which is the difference of the probability of dying in the group with the worst prognosis, and the group with the best prognosis. As described previously [21], we calculated PSEP at 3, 6, and 12 months after start of reirradiation.

Prognostic separation was also evaluated using the D-index, as developed by Royston and Sauerbrei [30]. The D-index can be interpreted as a robust version of the hazard ratio (HR) with 1 representing the null case, i.e. a difference in predicted survival does not result in a different observed survival. In the original article, it was reported as the logarithm of the HR (log-HR; 0 representing the null case) [30]. Different D-indices were compared using Student's t-test as described [31]. Discrimination (i.e. the ability of a model to correctly rank the patients by risk), was evaluated by calculating the Concordance-Index "C" as proposed by Harrell et al. [32]. C-index is an application of Somers' D and gives the probability that for a randomly chosen pair of patients, the predicted and the observed outcomes are concordant. A value of 0.5 indicates no predictive discrimination (i.e. the null case), and 1.0 indicates perfect separation of patients with different outcomes [21, 32, 33]. Different C-indices were compared as described by Kang et al. [34].

Finally we assessed the impact of each item with a Cox model, calculating the (Pseudo-) $\mathrm{R}^{2}$-coefficient according to Cox and Snell [35]. We included only items into multivariate modeling that were correlated with survival in univariate Kaplan-Meier models with a significance level of $p \leq 0.05$ (Mantel-Cox log-rank). All statistics were calculated using $\mathrm{R}$, a language and environment for statistical computing that is available for free online [31, 36]. Survival graphs, were prepared using SPSS Version 15 (SPSS Inc., Chicago IL).

\section{Results}

All reirradiations were applied as fractionated IMRTs. Median total dose was 30 Gy (10-45 Gy), delivered in 2 Gy fractions (range: 1.8-3.0 Gy). Reirradiation was discontinued in 4 patients due to worsening of general condition $(n=3)$ and death $(n=1)$. After a median follow-up of 36.2 months, 37 patients (84.1\%) had died. Median survival was 9 months after start of reirradiation (see Fig. 1a). Five patients (11.4\%) died within one month after their last fraction of reirradiation. We observed no case of RM or other severe late toxicities. One patient developed new sensory symptoms within 3 days after reirradiation but MRI showed no signs of RM, and symptoms resolved without intervention. Tumor control was 
Table 1 Patient characteristics of patients who received spinal reirradiation $(n=44)$

\begin{tabular}{|c|c|c|c|c|c|}
\hline \multirow{2}{*}{\multicolumn{2}{|c|}{ 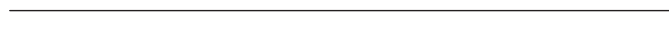 }} & \multirow{3}{*}{$\begin{array}{l}\text { Number of } \\
\text { patients } \\
21\end{array}$} & & & \\
\hline & & & \multirow[t]{2}{*}{ KPS at start [end] of reirradiation } & \multirow{2}{*}{$\begin{array}{l}\text { Median } \\
\text { Range }\end{array}$} & \multirow{2}{*}{$\begin{array}{l}70[70] \\
30-100 \\
{[20-100]}\end{array}$} \\
\hline \multirow[t]{2}{*}{ Sex } & Female & & & & \\
\hline & Male & 23 & \multirow{9}{*}{$\begin{array}{l}\text { Site of reirradiation [site of } \\
\text { first irradiation] }\end{array}$} & Cervical spine & $1[3]$ \\
\hline \multirow[t]{11}{*}{ Primary tumor site } & Breast & 17 & & Cervical and thoracic spine & $5[4]$ \\
\hline & Prostate & 9 & & Thoracic spine & $14[14]$ \\
\hline & $\begin{array}{l}\text { Kidney (renal cell } \\
\text { carcinoma) }\end{array}$ & 6 & & Thoracic and lumbar spine & $6[5]$ \\
\hline & Lung (non-small cell lung & 3 & & Lumbar spine & $10[10]$ \\
\hline & cancer) & & & Lumbar spine and os & $4[6]$ \\
\hline & Rectum & 2 & & sacrum & \\
\hline & Esophagus & 2 & & Os sacrum & $2[2]$ \\
\hline & Head and Neck & 1 & & $\begin{array}{l}\text { Overlapping more than } \\
2 \text { areas }\end{array}$ & $2[0]$ \\
\hline & Thyroid & 1 & \multirow{3}{*}{$\begin{array}{l}\text { Period between } 1^{\text {st }} \text { irradiation } \\
\text { and reirradiation (same site, } \\
\text { in months) } \\
\end{array}$} & Median & 21 \\
\hline & Unknown primary & 1 & & Range & $4-120$ \\
\hline & Sarcoma & 1 & & & \\
\hline
\end{tabular}

Number of spinal metastases

Pancreas

$n=1$

$n=2$

$n \geq 3$

Palsy

No

Incomplete

Complete

Number of extraspinal bone metastases

Visceral metastases

Liver metastases

Lung metastases

Brain metastases

Pleural effusion presentation

Age at start of reirradiation $n=0$

$n=1$

$n=2$

$n \geq 3$

No

Yes

Potentially removable

No

Yes

No

Yes

No

Yes

No

Yes

Unknown (no examination documented)

$\begin{array}{ll}\text { No } & 26 \\ \text { Yes } & 9 \\ \text { Unknown } & 9\end{array}$

Median (range)
Table 1 Patient characteristics of patients who received spinal reirradiation $(n=44)$ (Continued)

acceptable with 9 patients (20.5\%) developing local recurrences (LR). LR occurred at a median time of 12.2 months after initiation of reirradiation. Two cases of early recurrence within 3 months were observed, both were accompanied by rapid systemic tumor progression and death within 5 months. Local control (LC) as defined by diagnostic imaging or clinical examination was maintained until death or end of follow-up in 29 patients (65.9\%). LC was undetermined in 6 patients (13.6\%), including one patient who died during reirradiation and 2 patients who discontinued reirradiation.

Patients were classified into predictive groups as described by each SPT. SPT-Nieder contains a pleural effusion (PE) item and a steroid use item (see Additional file 1: Table S1). Scores of patients with unknown pleural status were assigned to the group with no PE, i.e. no routine thorax imaging was performed when PE was not suspected. The same applies to the steroid usage item: in some patients (timing of) steroid use was not specifically documented, these patients were assigned to the group without steroid use. To avoid bias, we re-calculated all SPT-Nieder results after excluding all patients with unknown pleural status, or specific documentation of steroid use $(n=11)$. As detailed below, this did not change results significantly. PSEP showed good prognostic separation, at months 3, 6, and 12 using SPT-Nieder (73.3-86.7\%). Good late separation after 12 months was also obtained applying SPT-Chow (70 \%), and SPT-Tokuhashi (64 \%), however both SPTs showed weak early results in months 3 and 6. All other SPTs showed insufficient separation as measured by PSEP (see Table 2). Calculation of C-indices showed that reasonable separation of prognostic groups can be obtained using SPT-Nieder $(C=0.68, p<0.001)$. Results were similar, if patients with unknown pleural 

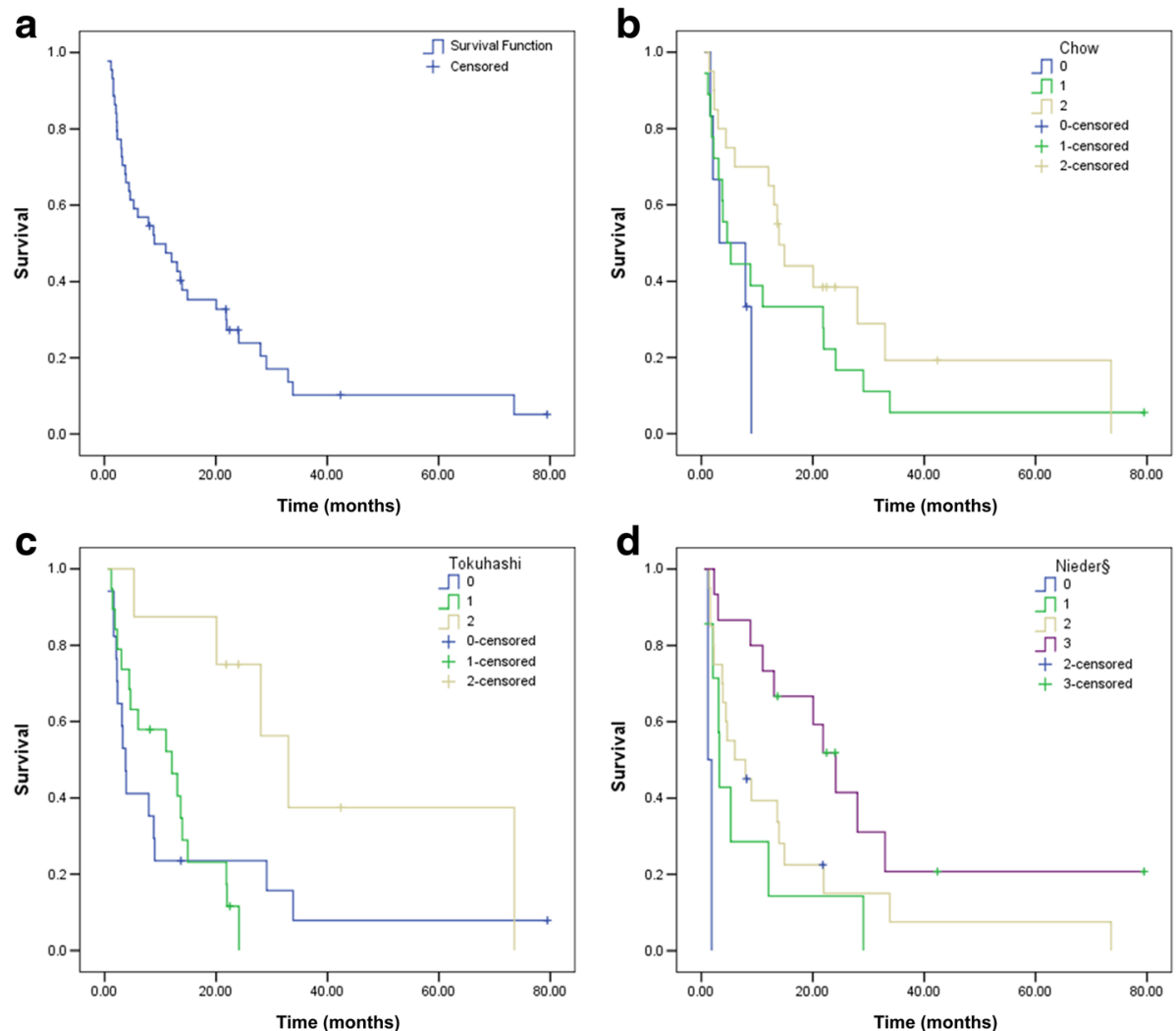

Fig. 1 Survival curves are shown for all patients in (a), median OS in all patients was 9 months. b-d show survival curves in patients assigned to risk groups as detailed in each SPT that showed prognostic separation as measured by PSEP, C-index, and D-index. Median OS in the following legend is ranked from best to worst predictive group. $\mathbf{b}$ Chow, median OS: 13.9/4.6/3.2 months, $p=0.11$. c Tokuhashi, median OS: 33/12.1/3.7 months, $p=0.033$. d Nieder, median OS: 24.1/6/3.2/1.2 months, $p<0.001 ; \S=$ patients with unknown PE and steroid use ranked as no PE and no steroid use

status were excluded $(\mathrm{C}=0.7, p<0.001)$. SPT-Tokuhashi, SPT-Bollen, SPT-Tomita, and SPT-Chow also showed significant but numerically worse prognostic separation $(\mathrm{C}=0.64, \mathrm{C}=0.63, \mathrm{C}=0.6$, and $\mathrm{C}=0.6$, all $p<0.05)$. $\mathrm{C}$ indices of SPT-Bauer and SPT-Oswestry did not differ significantly from the null case. Results obtained by calculation of $\mathrm{D}$-indices demonstrated that actual risk of dying in our patient sample during follow-up was predicted by

Table 2 PSEP results after 3, 6, and 12 months

\begin{tabular}{llll}
\hline SPT & PSEP at 3 months & PSEP at 6 months & PSEP at 12 months \\
\hline Bollen & $15 \%$ & $15 \%$ & $35 \%$ \\
Tomita & $26.7 \%$ & $28.9 \%$ & $40 \%$ \\
Bauer & $<0 \%$ & $<0 \%$ & $4.4 \%$ \\
Chow & $13.3 \%$ & $25 \%$ & $70 \%$ \\
Tokuhashi $^{2}$ & $35.3 \%$ & $46.3 \%$ & $64 \%$ \\
Nieder $^{a}$ & $86.7 \%$ & $86.7 \%$ & $73.3 \%$ \\
Nieder $^{b}$ & $90 \%$ & $90 \%$ & $70 \%$ \\
\hline
\end{tabular}

Nieder ${ }^{\mathrm{a}}$ : Patients with unknown pleural status were assigned to the group without pleural effusion

Nieder ${ }^{\text {b: }}$ Patients with unknown pleural status were excluded
SPT-Tokuhashi ( $\mathrm{D}=0.61, p=0.03$, estimated $\mathrm{HR}=1.84)$, SPT-Chow $(\mathrm{D}=0.66, p=0.04$, estimated $\mathrm{HR}=1.94)$, and SPT-Nieder $(\mathrm{D}=1.1, p<0.001$; estimated $\mathrm{HR}=3.02)$. All other scoring systems were not statistically different from the null case. If compared directly with the other scoring systems, SPT-Nieder showed significantly better prognostic discrimination as compared to all indices besides SPTTokuhashi and SPT-Chow $(p=0.1$ and $p=0.11)$. Survival curves of all SPTs that showed acceptable results in PSEP, $\mathrm{C}$-index, and D-index are shown in Fig. 1, i.e. SPT-Chow (b), SPT-Tokuhashi (c), SPT-Nieder (d). All other survival curves are shown in Additional file 2: Figure S1b-e.

If patients with unknown PE and steroid use were excluded, SPT-Nieder performed numerically better $(\mathrm{D}=1.43, p<0.001$; estimated $\mathrm{HR}=4.17)$ and consistently showed significantly better prognostic separation if directly compared to all other SPTs $(p<0.05)$ besides SPT-Tokuhashi $(p=0.06)$; see Additional file 2: Figure S1a for survival curve. To examine which items contributed to the performance of SPTs, we analyzed each item of each instrument in univariate survival functions. If patients had liver metastases, they died significantly earlier $(p=0.006$, 
median survival 13 vs. 3.2 months). Visceral metastases in general were not significantly associated with survival. Further classification into removable vs. non-removable metastases also did not show a significant survival advantage for patients with removable vs. unremovable metastases. Karnofsky Performance Status (KPS) was significantly associated with survival $(p<0.001$, median survival times depended on cut-off values). Steroid use was also associated with worse outcome $(p=0.028$, median survival 13.6 vs. 3.1 months, significance was maintained after exclusion of all patients with undocumented steroid use: $p=$ 0.038). All other items, including primary tumor site were not significantly associated with survival. Based on these observations, we adapted a new score to our collective. Basically this new SPT is similar to SPT-Nieder but has only three items: KPS (10-70 \% vs. 80-100 \%), liver metastases (yes vs. no), and steroid use (yes vs. no). In the Cox model it showed higher $\mathrm{R}^{2}$ (0.248 vs. 0.237; $\max$ possible 0.994 in both cases), comparison of D- and Cindices did not show significant differences as compared to SPT-Nieder (see Additional file 2: Figure S1f for survival curve).

\section{Discussion}

In a recent survey [37], radiation oncologists reported to assess life expectancy in $91 \%$ of their palliative patient evaluations. Their estimates were inaccurately optimistic with an overestimation of survival in $67 \%$ of cases [37] which is in line with other physician's survival estimates in palliative cancer care [38-40]. Predicting survival in the setting of reirradiation might be even more difficult. Patient samples are inhomogeneous, and as indicated by the historical Mayo Clinic series [7], and reproduced in our study, there is a wide range of observed survival times (historical series: 1-51 months vs. 0.5-79(+) months in our series). Except for SPT-Tokuhashi, SPTs developed to estimate survival after primary treatment of spinal metastases showed worse performance as compared to SPT-Nieder. The same was true for the well established SPT-Chow which is generally accepted as a valid survival tool in palliative cancer care [20-22]. On the other hand, SPT-Nieder which was developed in a small $(n=87)$, inhomogeneous patient sample including reirradiations for brain, bone, and lung metastases, as well as primary tumors, among others [23], showed acceptable predictive separation.

Detailed analysis of all items of the different scores, and of other patient parameters showed that SPT-Nieder included all items that were significantly associated with survival in our patient group, i.e. general condition, liver metastases, and steroid usage. Pleural effusion was the only item in SPT-Nieder that was not associated with survival in our collective. Nevertheless, we recommend to use SPT-Nieder without modification, until there is confirmation in an independent collective, that the PE item can be eliminated without loss of predictive accuracy. Consistent with Nieder's observations, primary tumor site had no impact on survival in our patients. We emphasize that this item should be avoided as it seems to have limited accuracy in situations in which patients with aggressive tumors already lived longer than expected to experience in-field recurrence. Furthermore, new therapies, such as immunotherapeutic approaches might profoundly change prognosis in primary tumors traditionally considered to confer worse prognosis. Survival prediction is especially relevant in patients who live long enough to experience local recurrence and may require dose escalation. Interestingly, of 6 patients who developed late recurrences (11-59 months), SPT-Nieder would have ranked 4 in the best, and two in the second best (of 4) prognostic group. A potential score incorporating liver metastases, KPS, and steroid usage, which excludes the PE item would have assigned 5 patients to the long-term survivor group, and 1 patient to the second best group (of 4). All other scores ranked at least one long-term survivor in worst or second worst prognostic group. These results indicate that longterm survivors at risk for late recurrences who might benefit from dose escalation might be identified with appropriate prediction tools.

It is reassuring that despite the heterogeneous group of patients, the only survival tool which was developed for general reirradiation purposes worked best in our patient group. Our study has several shortcomings, it is a retrospective analysis in a small patient collective. Like other studies which include only patients who actually received radiotherapy, our study has an exclusion bias, i.e. patients who were not fit enough to undergo radiotherapy were not included in any analysis. This explains the low number of patients in worst prognostic groups, although many patients had late stage disease. Radiation therapy regimens in our study reflected clinical decisions involving estimated life expectancy, and other factors, but none of the SPTs were used at that time in our practice. Five patients $(\sim 11.4 \%)$ in our study received some fractions of reirradiation during their last month of life. If radiotherapy at end of life should be completely avoided is controversial, however, single fraction or short course treatments should be preferred. A rate of $11 \%$ irradiations during the last month of life might indicate overoptimistic clinical expectations as described previously [37].

\section{Conclusion}

Taken together, predicting survival in patients who undergo palliative reirradiation for spinal metastases is possible with acceptable discrimination. SPT-Nieder showed best prognostic accuracy, although the pleural 
effusion item did not correlate with survival in our group. Radiation oncologists typically estimate survival using performance status, overall metastatic burden, presence of central nervous system metastases, and primary cancer site [37]. Our data indicate that this approach should be reconsidered when it comes to reirradiation of spinal metastases: typical predictive factors such as primary tumor, central nervous system metastases, and overall metastatic burden may have limited efficacy as compared to KPS, liver metastases, and steroid use.

\section{Ethics approval}

The study was approved by the ethics committee of Heidelberg University, Medical Faculty Mannheim. Committees reference number: 2013-602N-MA.

\section{Additional files}

\begin{abstract}
Additional file 1: Table S1. All items that contribute to each SPT are shown and ranked according to the risk that has been assigned to each item by the authors of each SPT. (PDF $166 \mathrm{~kb}$ )
\end{abstract}

Additional file 2: Figure S1. Survival curves in patients assigned to risk groups as detailed in SPT-Nieder (\# = excluding all patients with unknown pleural status, and steroid use), SPT-Bollen, SPT-Tomita, SPT-Oswestry, and SPT-Bauer are shown in a-e. Figure S2f. shows performance of risk groups when ranked according to a score that was specifically adapted to the collective but has not yet been validated in an independent patient group. Median OS in the following legend is ranked from best to worst predictive group. a) Nieder, median OS: 21.8/4.6/3.1/1.2 months, $p<0.001 ; \#=$ patients with unknown PE and unknown steroid use excluded. b) Bollen, median OS: 13/14.9/3.8/7.9 months, $p=0.12$. c) Tomita, median OS: 20.1/2.2/7.9/3.8 months, $p=0.35$. d) Oswestry, median OS: 14.9/6/3.1/3.8/29.1 $p=0.1$ months. e) Bauer, median OS: $13 / 7.9 / 24.1$ months, $p=0.75$. f) Specifically adapted SPT, median OS: $24.1 / 8.97 / 3.2 / 1.84$ months, $p<0.001$ (TIF 4362 kb)

\section{Abbreviations}

HR: hazard ratio; IMRT: Intensity Modulated Radiation Therapy; KPS: Karnofsky Performance Status; LC: local control; LR: local recurrence; PE: pleural effusion; PSEP: prognostic separation index; RM: radiation myelopathy; SBRT: Stereotactic Body Radiation Therapy; SPT: survival prediction tool.

\section{Competing interests}

Dr. Buergy reports personal fees from Siemens AG, personal fees from NB Capital Research $\mathrm{GmbH}$, outside the submitted work; L. Siedlitzki has nothing to disclose; Dr. Boda-Heggemann reports personal fees from Elekta AB, Sweden, during the conduct of the study; Dr. Wenz reports grants, personal fees and non-financial support from Elekta, grants, personal fees and non-financial support from Carl Zeiss Meditec, outside the submitted work: Dr. Lohr reports grants and personal fees from Elekta AB, Sweden, grants, personal fees and other from IBA, personal fees from C-RAD, during the conduct of the study.

\section{Authors' contribution}

DB conceived the study, performed statistical analysis and drafted the manuscript. LS collected all patient-related data and helped to perform statistical analysis. JBH, FW, and FL provided assistance with data collection, design of the study and manuscript revisions. All authors read and approved the final manuscript.

\section{Acknowledgements}

The authors gratefully acknowledge all contributors of the $R$ Project for Statistical Computing for their great voluntary work. We want to address special thanks to Dr. Benjamin Haibe-Kains for his advice in interpretation of survcomp's output.

Funding

None.

Received: 22 January 2016 Accepted: 8 February 2016

Published online: 08 March 2016

\section{References}

1. Allemani C, Weir HK, Carreira H, Harewood R, Spika D, Wang XS, Bannon F, Ahn JV, Johnson CJ, Bonaventure A, et al. Global surveillance of cancer survival 1995-2009: analysis of individual data for 25,676,887 patients from 279 population-based registries in 67 countries (CONCORD-2). Lancet. 2015;385:977-1010.

2. Rades D, Rudat V, Veninga $T$, Stalpers $\sqcup$, Hoskin PJ, Schild SE. Prognostic factors for functional outcome and survival after reirradiation for in-field recurrences of metastatic spinal cord compression. Cancer. 2008;113:1090-6.

3. Chow E, Harris K, Fan G, Tsao M, Sze WM. Palliative radiotherapy trials for bone metastases: a systematic review. J Clin Oncol. 2007;25:1423-36.

4. Damast S, Wright J, Bilsky M, Hsu M, Zhang Z, Lovelock M, Cox B, Zatcky J, Yamada Y. Impact of dose on local failure rates after image-guided reirradiation of recurrent paraspinal metastases. Int J Radiat Oncol Biol Phys. 2011;81:819-26.

5. Hoebers F, Heemsbergen W, Moor S, Lopez M, Klop M, Tesselaar M, Rasch C. Reirradiation for head-and-neck cancer: delicate balance between effectiveness and toxicity. Int J Radiat Oncol Biol Phys. 2011;81:e111-118.

6. Hahn CA, Zhou SM, Raynor R, Tisch A, Light K, Shafman T, Wong T, Kirkpatrick J, Turkington T, Hollis D, Marks LB. Dose-dependent effects of radiation therapy on cerebral blood flow, metabolism, and neurocognitive dysfunction. Int J Radiat Oncol Biol Phys. 2009;73:1082-7.

7. Schiff D, Shaw EG, Cascino TL. Outcome after spinal reirradiation for malignant epidural spinal cord compression. Ann Neurol. 1995;37:583-9.

8. Tan BC, Khor TH. Radiation myelitis in carcinoma of the nasopharynx. Clin Radiol. 1969;20:329-31.

9. Delanian S, Lefaix JL, Pradat PF. Radiation-induced neuropathy in cancer survivors. Radiother Oncol. 2012;105:273-82.

10. Rades D, Stalpers $L$, Veninga T, Hoskin PJ. Spinal reirradiation after shortcourse RT for metastatic spinal cord compression. Int J Radiat Oncol Biol Phys. 2005;63:872-5.

11. Ryu S, Gorty S, Kazee AM, Bogart J, Hahn SS, Dalal PS, Chung CT, Sagerman $\mathrm{RH}$. 'Full dose' reirradiation of human cervical spinal cord. Am J Clin Oncol. 2000;23:29-31.

12. Grosu AL, Andratschke N, Nieder C, Molls M. Retreatment of the spinal cord with palliative radiotherapy. Int J Radiat Oncol Biol Phys. 2002;52:1288-92.

13. Kirkpatrick JP, van der Kogel AJ, Schultheiss TE. Radiation dose-volume effects in the spinal cord. Int J Radiat Oncol Biol Phys. 2010;76:S42-49.

14. Sahgal A, Weinberg V, Ma L, Chang E, Chao S, Muacevic A, Gorgulho A, Soltys S, Gerszten PC, Ryu S, et al. Probabilities of radiation myelopathy specific to stereotactic body radiation therapy to guide safe practice. Int J Radiat Oncol Biol Phys. 2013;85:341-7.

15. Corbin KS, Hellman S, Weichselbaum RR. Extracranial oligometastases: a subset of metastases curable with stereotactic radiotherapy. J Clin Oncol. 2013;31:1384-90.

16. Rades D, Conde AJ, Garcia R, Cacicedo J, Segedin B, Perpar A, Schild SE. A new instrument for estimation of survival in elderly patients irradiated for metastatic spinal cord compression from breast cancer. Radiat Oncol. 2015;10:173.

17. Rades D, Veninga T, Bajrovic A, Karstens JH, Schild SE. A validated scoring system to identify long-term survivors after radiotherapy for metastatic spinal cord compression. Strahlenther Onkol. 2013;189:462-6.

18. Rades D, Hueppe M, Schild SE. A score to identify patients with metastatic spinal cord compression who may be candidates for best supportive care. Cancer. 2013:119:897-903.

19. Westhoff PG, de Graeff A, Monninkhof EM, Bollen L, Dijkstra SP, van der Steen-Banasik EM, van Vulpen M, Leer JW, Marijnen CA, van der Linden YM, Dutch Bone Metastasis Study G. An easy tool to predict survival in patients receiving radiation therapy for painful bone metastases. Int J Radiat Oncol Biol Phys. 2014;90:739-47. 
20. Chow E, Fung K, Panzarella T, Bezjak A, Danjoux C, Tannock I. A predictive model for survival in metastatic cancer patients attending an outpatient palliative radiotherapy clinic. Int J Radiat Oncol Biol Phys. 2002;53:1291-302.

21. Chow E, Abdolell M, Panzarella T, Harris K, Bezjak A, Warde P, Tannock I. Predictive model for survival in patients with advanced cancer. J Clin Oncol. 2008;26:5863-9.

22. Angelo K, Dalhaug A, Pawinski A, Haukland E, Nieder C. Survival prediction score: a simple but age-dependent method predicting prognosis in patients undergoing palliative radiotherapy. ISRN Oncol. 2014;2014:912865.

23. Nieder C, Andratschke N, Angelo K, Haukland E, Grosu AL. Development of a score predicting survival after palliative reirradiation. J Oncol. 2014;2014:128240

24. Tokuhashi Y, Matsuzaki H, Oda H, Oshima M, Ryu J. A revised scoring system for preoperative evaluation of metastatic spine tumor prognosis. Spine (Phila Pa 1976). 2005;30:2186-91.

25. Balain B, Jaiswal A, Trivedi JM, Eisenstein SM, Kuiper JH, Jaffray DC. The Oswestry Risk Index: an aid in the treatment of metastatic disease of the spine. Bone Joint J. 2013;95-B:210-6.

26. Bauer HC, Wedin R. Survival after surgery for spinal and extremity metastases. Prognostication in 241 patients. Acta Orthop Scand. 1995;66:143-6.

27. Bollen $L$, van der Linden $Y M$, Pondaag W, Fiocco M, Pattynama BP, Marijnen CA, Nelissen RG, Peul WC, Dijkstra PD. Prognostic factors associated with survival in patients with symptomatic spinal bone metastases: a retrospective cohort study of 1,043 patients. Neuro Oncol. 2014;16:991-8.

28. Tomita K, Kawahara N, Kobayashi T, Yoshida A, Murakami H, Akamaru T. Surgical strategy for spinal metastases. Spine (Phila Pa 1976). 2001;26:298-306.

29. Altman DG, Royston P. What do we mean by validating a prognostic model? Stat Med. 2000;19:453-73.

30. Royston P, Sauerbrei W. A new measure of prognostic separation in survival data. Stat Med. 2004;23:723-48.

31. Haibe-Kains B, Desmedt C, Sotiriou C, Bontempi G. A comparative study of survival models for breast cancer prognostication based on microarray data: does a single gene beat them all? Bioinformatics. 2008;24:2200-8.

32. Harrell Jr FE, Lee KL, Mark DB. Multivariable prognostic models: issues in developing models, evaluating assumptions and adequacy, and measuring and reducing errors. Stat Med. 1996;15:361-87.

33. Pencina MJ, D'Agostino RB. Overall $C$ as a measure of discrimination in survival analysis: model specific population value and confidence interval estimation. Stat Med. 2004;23:2109-23.

34. Kang L, Chen W, Petrick NA, Gallas BD. Comparing two correlated C indices with right-censored survival outcome: a one-shot nonparametric approach. Stat Med. 2015;34:685-703.

35. Cox DR, Snell EJ. Analysis of binary data. 2nd ed. London: New York: Chapman and Hall; 1989.

36. R_Core_Team. R: A language and environment for statistical computing. Vienna, Austria: R foundation for Statistical Computing; 2015.

37. Tseng YD, Krishnan MS, Sullivan AJ, Jones JA, Chow E, Balboni TA. How radiation oncologists evaluate and incorporate life expectancy estimates into the treatment of palliative cancer patients: a survey-based study. Int J Radiat Oncol Biol Phys. 2013;87:471-8.

38. Chow E, Harth T, Hruby G, Finkelstein J, Wu J, Danjoux C. How accurate are physicians' clinical predictions of survival and the available prognostic tools in estimating survival times in terminally ill cancer patients? A systematic review. Clin Oncol (R Coll Radiol). 2001;13:209-18.

39. Christakis NA, Lamont EB. Extent and determinants of error in physicians' prognoses in terminally ill patients: prospective cohort study. West J Med. 2000;172:310-3.

40. Mackillop WJ, Quirt CF. Measuring the accuracy of prognostic judgments in oncology. J Clin Epidemiol. 1997;50:21-9.

\section{Submit your next manuscript to BioMed Central and we will help you at every step:}

- We accept pre-submission inquiries

- Our selector tool helps you to find the most relevant journal

- We provide round the clock customer support

- Convenient online submission

- Thorough peer review

- Inclusion in PubMed and all major indexing services

- Maximum visibility for your research

Submit your manuscript at www.biomedcentral.com/submit

) Biomed Central 